Cuad. Soc. Esp. Cienc. For. 46(1): 57-70 (2020)

Doi: https://doi.org/10.31167/csecfv0i46.19894

Sociedad Española

\title{
Primera aproximación al estudio de la actividad fisiológica en Quercus suber con alta presencia de Cerambyx welensi Küster
}

First approach to the study of the physiological activity of Quercus suber with high presence of Cerambyx welensi Küster

Sánchez-Osorio, I. ${ }^{1 *}$; López-Pantoja, G. '; Tapias, R. '; Pareja-Sánchez, E' ${ }^{1}$; Domínguez, L. ${ }^{1}$ 


\section{Resumen}

La incidencia de Cerambyx welensii en Quercus suber es un factor clave en el decaimiento de árboles en las dehesas. Las pautas de selección de hospedantes por $C$. welensii son poco conocidas. Entre otros factores, los volátiles de plantas se consideran claves en la localización de hospedantes en cerambícidos. En el presente trabajo estudiamos algunas características morfológicas de los árboles, así como la variación a corto plazo en la actividad fisiológica en $Q$. suber con distintos niveles de presencia de adultos de $C$. welensii. Nuestro objetivo fue analizar si la actividad fisiológica de $Q$. suber en condiciones de estrés veraniego podría variar entre árboles con distinta preferencia por $C$. welensii. Se estudiaron $36 Q$. suber (18 árboles muy frecuentados por $C$. welensii; 18 sin avistamientos del insecto), en los cuales se midió la fotosíntesis neta, conductancia estomática y transpiración durante un período previo e incluyendo el inicio de vuelo diario de $C$. welensii (19:00 - 21:20 h); se analizó además el perímetro y la superficie de proyección de copa de los árboles. Los árboles con $C$. welensii exhibieron tasas fotosintéticas más altas $(1.5-2.15$ veces $)$ que los árboles sin avistamientos del insecto entre las 19:35 y las 20:45, así como mayor perímetro y menor cociente superficie de proyección de copa a perímetro. Los resultados sugieren que tanto los ajustes fisiológicos bajo estrés ambiental (influyendo en el patrón de emisión de monoterpenos) como las características morfológicas de los árboles podrían tener un efecto en la selección intraespecífica de hospedantes por $C$. welensii.

Palabras clave: Cerambycidae, conductancia estomática, dehesa, fotosíntesis, transpiración, xilófago.

\section{Summary}

The incidence of Cerambyx welensii on Quercus suber is a key factor in the decline of trees in dehesa woodlands. Plant volatiles are important in plant-insect interactions, but the host-plant selection mechanism used by $C$. welensii remains unknown. We studied the short-term variations in physiological performance in $Q$. suber trees highly visited by $C$. welensii, as well as some morphological features. We hypothesized that physiological adaptation by $Q$. suber to cope with stress conditions could influence monoterpene emission patterns, thereby affecting intra-specific host-selection cues used by $C$. welensii. Altogether 36 Q. suber trees (18 with high presence of adults of $C$. welensii and 18 without presence of this cerambycid species) were studied for physiological performance (photosynthesis, stomatal conductance and transpiration), as well as perimeter and crown silhouette area, during a period prior to and including the start time of $C$. welensii flight activity $(19: 00-21: 20 \mathrm{~h})$. The trees with presence of $C$. welensii trended to exhibit higher $(1.5-2.15$ times $)$ photosynthetic values from 19:35 to 20:45, as well as larger perimeter than trees without $C$. welensii. The results suggest that both some morphological features (in a direct way, via visual cues) as well as short-term physiological adjustments under environmental stress (in a indirect way, by its effect in the monoterpene emission pattern), could affect intra-specific host selection by $C$. welensii.

Keywords: Cerambycidae, open woodland, photosynthesis, stomatal conductance, transpiration, xylophagous. 


\section{Introducción}

La dehesa es un sistema de aprovechamiento agrosilvopastoral que, bien llevado a la práctica, supondría un uso sostenible de los recursos naturales, en armonía con el mantenimiento de unos altos valores de biodiversidad biológica. Desde el punto de vista socioeconómico, las dehesas han permitido durante siglos el desarrollo social de ciertas zonas tradicionalmente desfavorecidas, por ello, estos sistemas se citan como ejemplo modélico de sistema agrario de Alto Valor Natural (AVN) (WWF-Adena, 2006). Los sistemas AVN constituyen una pieza clave del Plan de Acción sobre la Biodiversidad de la UE (Comisón Europea, 2008) y de la Estrategia Sobre la Diversidad Biológica y Paisajística del Consejo de Europa. Tanto las directrices políticas europeas en materia de desarrollo rural como las nacionales, han considerado prioritarias las líneas de investigación sobre la dehesa, en particular aquellas aplicadas al control integrado de plagas del arbolado de las dehesas (Consejo de las Comunidades Europeas, 1992; Comunidad Autónoma de Andalucía, 2010). Las formaciones de Quercus suber L. están sometidas a múltiples factores de estrés (Aronson et al., 2009), tanto antropogénicos como naturales. Entre ellos, la actividad del cerambícido xilófago Cerambyx welensii Küster (Coleoptera, Cerambycidae) se considera un factor clave en el decaimiento de $Q$. suber en el suroeste español (López-Pantoja et al., 2008; Torres-Vila et al., 2017).

Cerambyx welensii es un insecto xilófago de gran tamaño (hasta $60 \mathrm{~mm}$ de longitud) que afecta a diversos géneros arbóreos caducifolios y esclerófilos, principalmente al género Quercus (Vives, 2000). Su actividad de vuelo se desarrolla durante el verano, presentando los adultos una actividad diaria fundamentalmente crepuscular (López-Pantoja et al., 2008; datos de los autores). El daño causado por $C$. welensii se debe exclusivamente a su actividad larvaria, que origina la ruptura de ramas y troncos de los árboles (López-Pantoja et al., 2008) y facilita la infección por patógenos y hongos de pudrición (Martín et al., 2005). La repercusión de este insecto puede llegar a ser preocupante. Así, se ha llegado a citar en Extremadura un grado de colonización de $Q$. suber por $C$. welensii superior al $34 \%$ de los árboles (Vicente, 2013).

Los terpenoides y otros compuestos orgánicos volátiles desempeñan un papel importante tanto en la fisiología de las plantas como en las interacciones de las plantas con su entorno, por ejemplo, protegiendo las plantas contra los factores de estrés o actuando como un mecanismo para la comunicación entre individuos (Grote et al., 2013; Loreto et al., 2014). El género Quercus ha sido reconocido entre los mayores emisores de compuestos orgánicos volátiles (COV) (Pearse et al., 2013), considerándose Q. suber como un fuerte emisor de monoterpenos (Pio et al., 2005; Lavoir et al., 2011). Existe considerable evidencia de que los cerambícidos son atraídos por compuestos volátiles como los monoterpenos, estando los compuestos volátiles probablemente involucrados en el reconocimiento de huéspedes debilitados y dañados (Allison et al., 2004). Recientemente se ha asociado una mayor presencia de daños y adultos de $C$. welensii en $Q$. suber con perfil de emisión foliar tipo limoneno que en árboles con perfil de tipo pineno (Sánchez-Osorio et al., 2019).

El estrés ambiental severo, como ocurre en el verano en las áreas mediterráne- 
as, induce cambios fisiológicos y bioquímicos en las plantas, y la fotosíntesis se considera una de las funciones celulares más sensibles a las altas temperaturas y la sequía severa (Correia et al., 2014; Piayda et al., 2014). En Q. suber se han citado disminuciones reseñables tanto de la conductancia estomática $(37 \%)$ como de la tasa máxima de carboxilación (43\%) bajo sequía severa (Piayda et al., 2014). En Pinus halepensis Mill., bajas tasas de fotosíntesis y conductancia estomática medidas en entornos climáticos extremos se han considerado un indicador de éxito adaptativo al estrés severo (Llusià et al., 2015). Factores ambientales como la temperatura y la radiación fotosintéticamente activa están entre los más condicionantes de la emisión de COV a corto plazo (Hakola et al., 2001), puesto que los precursores de monoterpenos derivan de la actividad fotosintética (Loreto et al., 1996). Peñuelas y Llusià (1999) encontraron correlaciones significativas de las tasas diarias de emisión de terpenos (especialmente de limoneno, el monoterpeno menos volátil) con tasas fotosintéticas netas en Quercus ilex L. en condiciones de verano, pero correlaciones más débiles con la conductancia estomática, como también encontraron Loreto et al. (1996). En relación con lo anterior, Lavoir et al. (2005) encontraron una mayor eficiencia del fotosistema II bajo fotoestrés en hojas $Q$. suber que en hojas de $Q$. ilex en condiciones de alta y baja temperatura.

Este estudio pretende contribuir al conocimiento de los mecanismos que condicionan la selección de hospedantes por parte de $C$. welensii, en particular la relación entre variables fisiológicas y morfológicas del arbolado y la emisión de compuestos orgánicos volátiles que podrían participar en la localización olfativa de ejemplares de $Q$. suber. Los objetivos que se han planteado son los siguientes:

1. Analizar la variación a corto plazo de la fotosíntesis neta, conductividad estomática y transpiración en árboles con distinto grado de presencia de $C$. welensii.

2. Comparar la morfología de árboles con distinto perfil de emisión de monoterpenos foliares.

3. Estudiar la relación entre las características morfofisiológicas de los árboles y su perfil de emisión de monoterpenos foliares.

\section{Material y Métodos}

\section{1. Árboles estudiados}

El estudio se realizó el año 2008, en la dehesa San Enrique (Almonte, Huelva. $37^{\circ} 15^{\prime} 43.73$ "N, $6^{\circ} 28^{\prime} 34.65^{\prime \prime} \mathrm{O}, 80 \mathrm{~m} \mathrm{snm}$ ) fuertemente afectada por $C$. welensii, y poblada principalmente por $Q$. suber (densidad de 75 árboles ha ${ }^{-1}$ ). La temperatura media anual fue de $17,6^{\circ} \mathrm{C} \pm 0,2$, y la precipitación anual fue de $588 \mathrm{~mm}$ (estación de Almonte). Se estudió un total de 36 árboles, escogidos a partir de los avistamientos de $C$. welensii computados en cada árbol entre 2002 y 2007 
(López-Pantoja et al., 2008), y repartidos en dos grupos de 18 árboles cada uno. E1 primer grupo, de aquí en adelante llamados árboles con $C$. welensii, incluyó aquellos con más visitas registradas de individuos diferentes (promedio de $54 \pm 23$ insectos por árbol entre 2002 y 2007); estos árboles también mostraron el mayor grado de daño por grandes perforadores de leño. El segundo grupo, en adelante llamado árboles $\sin C$. welensii, comprendía pies de $Q$. suber contiguos a los árboles visitados, pero en los que no se registró avistamiento alguno de $C$. welensii ni daños atribuibles a este perforador durante los años referidos. Este comportamiento de los árboles en lo relativo a la presencia de adultos de $C$. welensii se verificó en 2008, en muestreos realizados las semanas previas a la toma de datos propias de este trabajo.

\subsection{Medición de variables}

En los árboles escogidos se midieron in situ la tasa fotosintética (asimilación neta de $\left.\mathrm{CO}_{2}, \mathrm{~A}\right)$, conductancia estomática $(g s)$ y transpiración $(E)$. Las medidas de $A$, gs y $E$ se realizaron mediante un analizador de gases por infrarrojo (IRGA) con cámara de hoja ancha $\left(6.25 \mathrm{~cm}^{2}\right.$; ADC Scientifics Ltd, Modelo Lci, Reino Unido). Para ello se recolectó una rama de cada árbol $(\approx 2 \mathrm{~m}$ de altura en exposición de sol, con $30 \mathrm{~cm}$ de largo, 53-187 hojas y peso seco medio de $14.48 \pm 0.7 \mathrm{~g}$ ), efectuando inmediatamente después el muestreo de monoterpenos foliares (ver Sánchez-Osorio et al., 2019 para más detalles; datos no incluidos en este trabajo) y las mediciones de $A, g s$ y $E$ s a partir de una hoja por rama, perteneciente al brote del año.

La temperatura, la humedad relativa y la radiación fotosintéticamente activa $(P P F D)$ también se anotaron durante el transcurso de las mediciones. Las mediciones se efectuaron en una fecha cercana al pico estimado del período de vuelo anual de $C$. welensii (24 de junio. López-Pantoja et al., 2008), durante un intervalo de tiempo que incluía el período anterior al inicio del vuelo diario de $C$. welensii, así como el propio inicio de la actividad diaria de vuelo del insecto. Para ello se establecieron cinco rondas de medición (19:00, 19:35, 20:10, 20:45 y 21:20 horas); en cada ronda se realizaron mediciones en grupos de tres o cuatro árboles tomados aleatoriamente de cada tipología (con / $\sin C$. welensii), comprendiendo cada ronda un grupo distinto de árboles.

Además de las tres variables fisiológicas, se tomaron datos de algunas características morfológicas de los árboles que podrían tener relación con su actividad fisiológica y/o con la selección por parte de $C$. welensii. Por un lado se midió en todos los árboles el perímetro a la altura del pecho. Por otro lado, se utilizaron imágenes satélite de alta resolución (Google, 2007) para determinar la superficie de proyección de la copa $(S P C)$ de los árboles, para lo cual se crearon cuidadosamente con ayuda de ArcGis 10.0 polígonos que representaban el área de la proyección vertical de la copa de cada árbol.

\subsection{Análisis estadístico}

Los análisis comparativos para cada una de las variables fisiológicas se realiza- 
ron utilizando modelos lineales mixtos (MLM, paquete lme4; Bates et al., 2014). La variable dependiente fue, en cada caso, la medición promedio; como factores fijos se emplearon la presencia de $C$. welensii y la ronda temporal de medición (variable tiempo). La identidad del árbol (modelo de intercepción) se utilizó como factor aleatorio. La importancia de los factores principales se evaluó mediante el análisis de las razones de verosimilitud. Para los contrastes múltiples, los $P$-valores se ajustaron según el procedimiento de Benjamini and Hochberg (1995) (paquete Lsmeans; Lenth, 2014).

Para analizar la posible influencia de la actividad fisiológica y las características morfológicas de los árboles sobre las emisiones de monoterpenos foliares, se realizó un análisis de correspondencia canónica parcial (pCCA, paquete vegan; Oksanen et al., 2015), con la variable perímetro tomada como covariable. Para este propósito, los datos fueron estandarizados (a media cero y varianza unidad) en cada ronda de mediciones. La multicolinealidad se analizó examinando los factores de inflación de la varianza. Las variables explicativas integrantes del modelo final fueron: fotosíntesis neta $(A)$, transpiración $(E)$ y la relación entre el área de la silueta de la proyección de copa y el perímetro ( $S P C$ / perímetro). Las variables respuesta fueron: emisiones relativas individuales (porcentajes sobre la emisión conjunta de $\alpha$-pineno, $\beta$-pineno, sabineno, limoneno y mirceno) para limoneno y mirceno, así como una nueva variable creada (llamada tipo pineno) correspondiente a la suma de las emisiones relativas del $\alpha$-pineno, $\beta$-pineno y sabineno. La importancia de los ejes canónicos se evaluó con pruebas de permutación utilizando el paquete vegan del programa R.

Los análisis estadísticos se realizaron con el programa $\mathrm{R}$, versión 3.1.0, utilizando $\alpha=0.05$ y $\alpha=0.1$ como umbrales de significación y significación marginal.

\section{Resultados}

La temperatura del aire $(T)$ fue alta durante las rondas de medición de las variables fisiológicas $\left(>28.1^{\circ} \mathrm{C}\right)$. La humedad relativa $(H R)$ fue muy baja $(<40 \%$ en general); además, la PPFD disminuyó drásticamente con el transcurso de las rondas

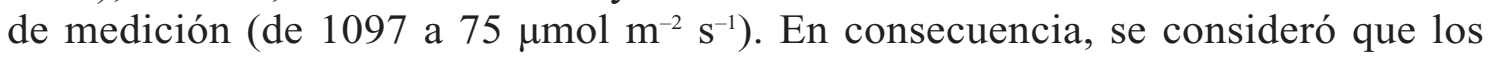
datos obtenidos para las tres variables fisiológicas $(A, g s$ y $E)$ en la ronda de las 21:20 h no presentaban suficiente fiabilidad, y por lo tanto esos datos no se analizaron y no se muestran (Figura $1 b-d$ ).

No encontramos correlaciones significativas entre $A$ y $E$ o $g s$, pero se encontró una correlación positiva muy significativa $(P<0.01)$ entre los valores estandarizados de $E$ y $g s$, tanto en los árboles $\sin C$. welensii $(\mathrm{R}=0.61)$ como en aquellos con C. welensii $(\mathrm{R}=0.82)$. Entre estas variables, sólo la fotosíntesis neta mostró cambios significativos a corto plazo durante las rondas de medición (Figura 1), con un efecto significativo de la interacción tiempo x presencia en las tasas de $A$ (MLM: $\chi 23=9.83, P<0.01)$. Específicamente, los valores A en árboles $\sin C$. welensii excedieron a los encontrados en árboles con presencia de $C$. welensii a las 19:00 $(\mathrm{P}=$ 0.08; Figura $1 b$ ), después de lo cual las tasas de $A$ en árboles con $C$. welensii alcan- 

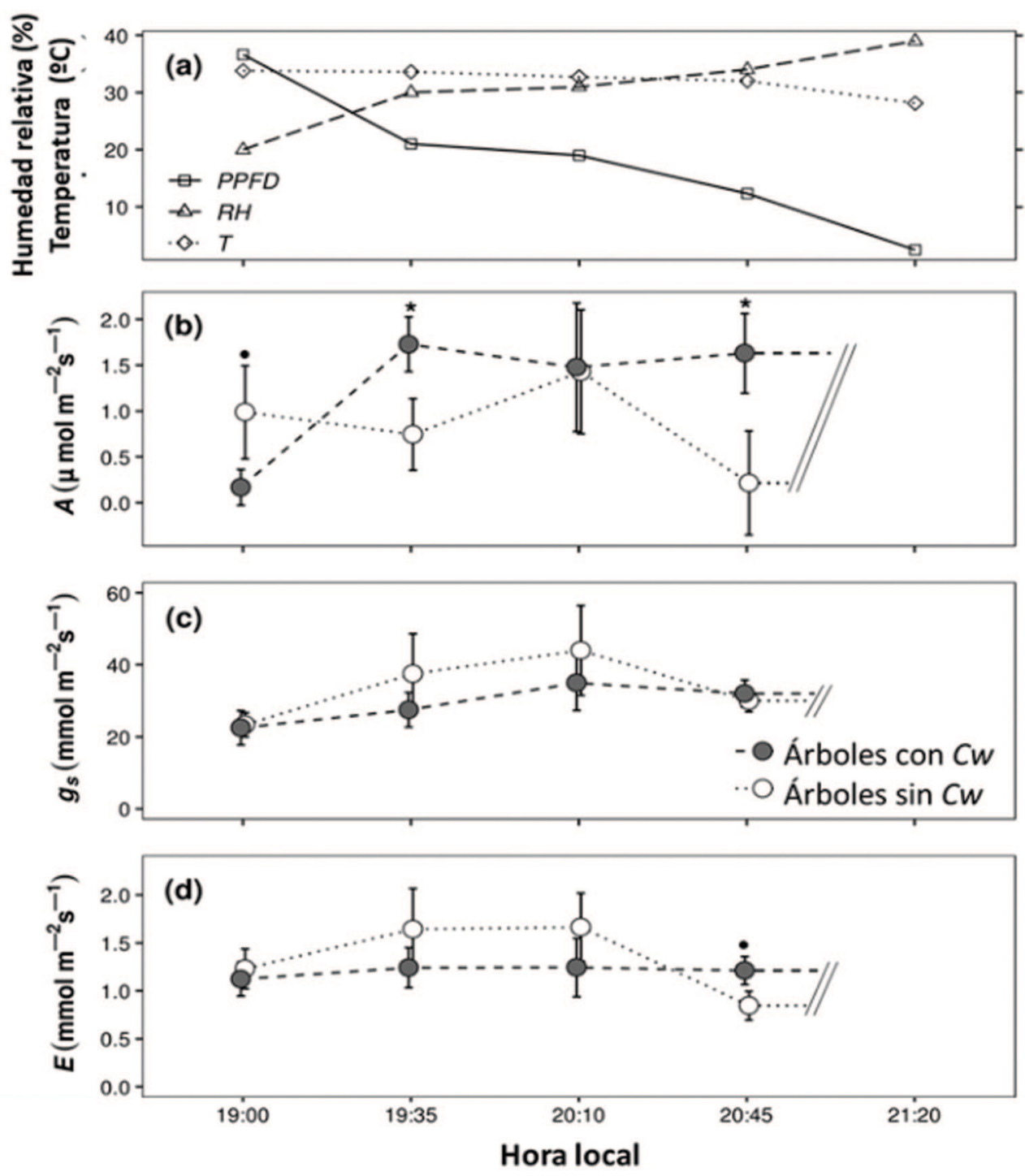

Figura 1. (a) Temperatura del aire $(T)$, humedad relativa $(H R)$ y densidad de flujo de fotones fotosintéticos (PPFD) en Quercus suber en 2008; (b) fotosíntesis $(A)$, (c) conductancia estomática $(g s)$ y $(\mathrm{d})$ transpiración $(E)$ de $Q$. suber estratificada según los árboles tuvieran o no presencia de Cerambyx welensii. Para cada tiempo de medición, un símbolo sobre el valor medio $( \pm \mathrm{EE})$ denota diferencias significativas entre árboles con distinto estatus de presencia de $C$. welensii. ( ${ }^{*}, P<0.05 ; \bullet, \mathrm{P}<0.1$. MLM con corrección de Benjamini-Hochberg. $\mathrm{N}=3-4$ por ronda de muestreo para cada grupo de árboles estudiado). No se muestran los datos para $A$, gs y $E$ medidos a las 21:20 h.

zaron valores más altos que los medidos en árboles sin presencia de $\mathrm{C}$. welensii a las 19:35 $(\mathrm{P}=0.04)$ y a las $20: 45(\mathrm{P}=0.03)$.

Los árboles con $C$. welensii presentaron un perímetro medio significativamente mayor que los árboles sin $C$. welensii $(168.4$ y $131.1 \mathrm{~cm}$, respectivamente; $P=$ 0.01 ). Los valores de $S P C$ fueron similares en ambos grupos de árboles (49.2 y 47.8 $\mathrm{m}^{2}$, para árboles con y $\sin C$. welensii respectivamente), pero los primeros tuvieron 
una relación $S P C$ / perímetro más baja (30.7 y 36.3 m respectivamente. $P=0.2)$. Para los árboles sin $C$. welensii el modelo pCCA no fue significativo (prueba de permutación: $P=0.38$; datos no mostrados). En contraste, los primeros dos ejes del modelo pCCA para el conjunto de árboles, tanto con presencia como con ausencia de adultos de $C$. welensii, permitieron un explicación baja y marginalmente significativa $(19 \%, P=0.07)$ de la varianza correspondiente a las emisiones relativas de limoneno, mirceno y de la nueva variable tipo pineno (Figura $2 a$ ); pero dicho modelo dio mejores resultados cuando se aplicó sólo a los árboles con presencia de $C$. welensii (62\% de varianza explicada, $P=0.001)$ (Figura $2 b)$.
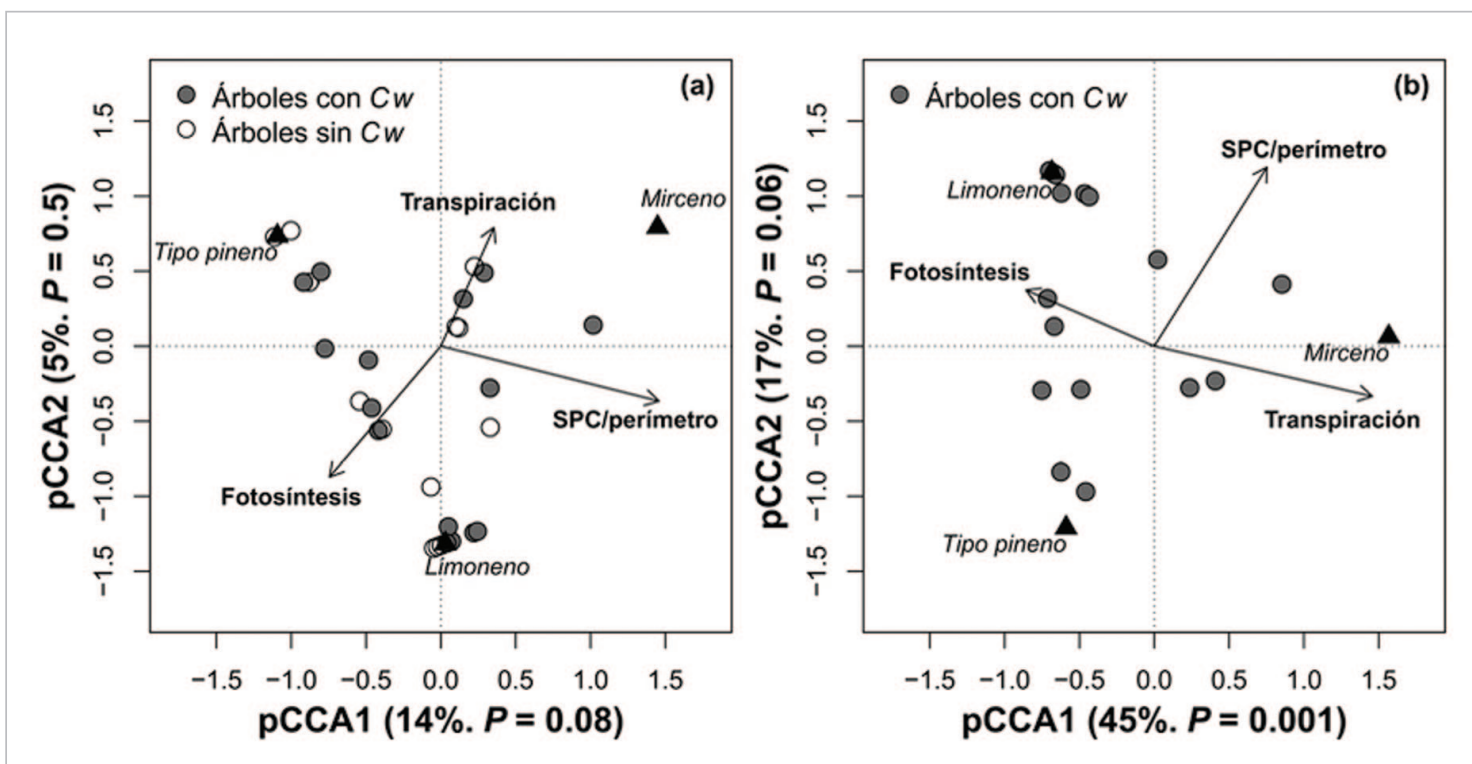

Figura 2. Gráficos triplot correspondientes al análisis de correspondencia canónica parcial (Scaling 1) para: (a) todos los ejemplares de Quercus suber estudiados y (b) ejemplares de $Q$. suber con elevada presencia de adultos de Cerambyx welensii $(C w)$. El gráfico ilustra el efecto de las variables fisiológicas (fotosíntesis, transpiración y conductancia estomática), así como la razón entre la superficie de proyección de la copa y el perímetro del tronco (SPC / perímetro) sobre la composición de las emisiones (emisiones relativas) representadas por limoneno, mirceno y la variable tipo pineno (emisión relativa conjunta de $\alpha$ - pineno, $\beta$ - pineno y sabineno). Los triángulos representan las puntuaciones de las variables representativas de la emisión relativa.

Para los árboles con $C$. welensii el eje pCCA1 resultó significativo $(P<0.002)$ mientras que el eje pCCA2 fue marginalmente significativo $(P=0.06)$; en cambio, sólo el eje pCCA1 fue significativo (marginalmente) para el modelo aplicado al conjunto de árboles (Figura 1a). El eje pCCA1 se correlacionó principalmente con la fotosíntesis y secundariamente con la transpiración en el grupo de árboles con $C$. welensii (puntuaciones biplot: -0.46 y 0.42 , respectivamente), mientras que para el conjunto de árboles dicho eje se vinculó principalmente a la variable morfológica $S P C$ / perímetro (puntuación biplot: 0.17). El eje pCCA2 se correlacionó, en el grupo de árboles con $C$. welensii, principalmente con la razón $S P C$ / perímetro (pun- 
tuación biplot: 0.41). La variable limoneno tuvo puntuaciones medias (valor absoluto de 0.57 ) en ambos ejes pCCA en el modelo para árboles con $C$. welensii; en contraste, la variable tipo pineno presentó una puntuación baja en pCCA1 (-0.16) pero un valor medio en pCCA2 (0.63).

En resumen, el análisis de los modelos sugiere que los árboles con presencia de C. welensii en los que la emisión relativa de limoneno fue mayor son aquellos con los valores más altos de fotosíntesis y razón $S P C$ / perímetro, junto con valores bajos de transpiración. En contraste, los árboles con presencia de $C$. welensii que tuvieron las emisiones relativas más altas de compuestos de tipo pineno fueron aquellos con valores de $S P C$ / perímetro inferiores al promedio, siendo menos claro en estos árboles el efecto de la fotosíntesis y la transpiración (Figura 2). El análisis realizado sobre el conjunto de árboles (con y sin presencia de $C$. welensii) deparó una explicación mucho menos clara de la influencia de las variables morfo-fisiológicas en la emisión. Así, en este caso parece sugerirse una ligera asociación general entre la razón $S P C$ / perímetro y la emisión de limoneno; pero dicha variable morfológica mostró correlación inversa con la tasa de emisión de compuestos tipo pineno, para lo que no encontramos una explicación evidente.

\section{Discusión}

En el ámbito mediterráneo, el estrés por sequía, elevadas temperaturas y alta insolación puede originar, en especies representativas como $Q$. suber y $Q$. ilex, situaciones de estrés oxidativo y disminución en la tasa fotosintética asociada a la disminución de la conductancia estomática (Munné et al., 2004 y trabajos citados allí; Vaz et al., 2010). La resiliencia tras episodios de estrés supone un rasgo adaptativo de los árboles ante condiciones de crecimiento con limitación en la disponibilidad de agua (Vaz et al., 2011). En estos contextos, se ha citado que la emisión constitutiva de compuestos volátiles como los monoterpenos, y en particular el limoneno, podría participar en la tolerancia al estrés abiótico —en particular al estrés térmico veraniego-, al menos en condiciones como la ausencia de fotorrespiración (Peñuelas y Llusià, 2002; Llusià et al., 2005; Niinemets and Monson, 2013; Loreto et al., 2014).

A lo largo de las cinco rondas de medición, en nuestro estudio se encontraron valores bajos para las variables fisiológicas $A, g s$ y $E$, que resultaron estar, no obstante, dentro de los rangos ya citados previamente para $Q$. suber y $Q$. ilex en similares condiciones de medición (Tenhunen et al., 1989; Oliveira et al., 1993; Niinemets et al., 2002). Se ha encontrado que temperaturas del aire elevadas $\left(>25^{\circ} \mathrm{C}\right)$ reducen o inhiben la tasa fotosintética en $Q$. suber y $Q$. ilex (Delfine et al., 2000; Peñuelas et al., 2005). La temperatura encontrada durante nuestras mediciones, unida probablemente a una pobre disponibilidad de agua en el suelo, podría haber tenido un efecto negativo en las tasas de $A$, en consonancia con lo recogido por Hakola et al., (2001) y Núñez et al. (2002). Pese a estos valores generales moderados de asimilación neta de $\mathrm{CO}_{2}$, esta tasa resultó significativamente diferente entre árboles con presencia de adultos de $C$. welensii y árboles sin presencia del insecto. 
Las pautas de selección de hospedantes por cerambícidos xilófagos que afectan a frondosas siguen siendo poco conocidas. Los compuestos volátiles liberados por plantas dañadas o estresadas pueden proporcionar señales olfativas que los cerambícidos usan para localizar hospedantes adecuados para sus larvas (Allison et al. 2004; Millar and Hanks, 2017). Se ha sugerido, además, que las señales visuales también podrían influir en la localización de árboles por $C$. welensii (Torres-Vila et al., 2017). En la dehesa de nuestro estudio, López-Pantoja et al. (2008) encontraron una presencia relevante de individuos de $C$. welensii transeúntes, sugiriendo que alguna pauta de selección de hospedantes podría mediar estos movimientos entre áreas cercanas. En nuestro estudio, los árboles con $C$. welensii tuvieron un perímetro medio significativamente mayor, pero una proporción menor (no significativa) de la razón de la superficie de proyección de copa al perímetro del tronco que los árboles $\sin C$. welensii. Este resultado parece estar en consonancia con la preferencia de $C$. welensii por árboles viejos y con cierto grado de deterioro (López-Pantoja et al., 2008).

Cerambyx welensii muestra sensibilidad antenal al limoneno, mirceno y compuestos de tipo pineno (Sánchez-Osorio, 2005). Se han encontrado evidencias de la existencia de diferencias en las emisiones foliares de monoterpenos entre $Q$. suber con alta presencia de adultos de $C$. welensii y árboles vecinos sin presencia de este insecto, resultando tener el limoneno una alta relevancia en la composición monoterpénica en árboles con $C$. welensii, mientras que en árboles sin presencia de adultos de esta especie predominaron los compuestos de tipo pineno. Sin embargo, se ha encontrado una baja atracción de $C$. welensii hacia trampas cebadas con monoterpenos únicamente (en particular, $\beta$-pineno) en comparación con la mayor eficacia de los cebos a base de mezclas sintéticas imitadoras de material vegetal fermentado (Sánchez-Osorio et al., 2016). Tanto el modelo pCCA significativo obtenido para el grupo de árboles con presencia de $C$. welensii como las diferencias en la asimilación neta de $\mathrm{CO}_{2}$ encontradas entre los dos grupos de árboles estudiados sugieren que, de acuerdo con Pio et al. (2005), el mantenimiento de la actividad fotosintética durante las horas de la tarde previas al crepúsculo podría haber favorecido la emisión posterior, precisamente en momentos en el que los adultos de $C$. welensii inician su actividad diaria, del monoterpeno menos volátil (limoneno).

Una infestación severa por $C$. welensii en árboles viejos aumenta el riesgo de cambios en la arquitectura de la copa (es decir, cambios en la relación $S P C$ / perímetro), ya que la actividad larvaria causa la rotura de ramas y troncos. En nuestro estudio, la relación $S P C$ / perímetro de árboles con $C$. welensii fue un $14 \%$ más pequeña que la de los árboles $\sin C$. welensii. Este cambio no fue significativo, pero abre un interesante punto de estudio ya que son prácticas selvícolas habituales en alcornocales las podas de grandes ramas afectadas por insectos xilófagos, lo que podría disminuir en esos árboles la relación $S P C$ / perímetro. Las alteraciones en la arquitectura de la copa podrían modificar la silueta de los árboles, influyendo potencialmente en la elección del huésped por la vía de señales visuales (Torres-Vila et al., 2017). Pero dicha alteración también podría influir (a través de cambios en la intercepción de la luz y/o la conductancia hidráulica) tanto en la actividad fisiológica y el comportamiento de las emisiones de compuestos volátiles foliares (Grote, 
2007), como en la densidad de la madera (lo que influiría en la idoneidad de los árboles para la supervivencia de las progenies de C. welensii (Hoeber et al., 2014)).

\section{Conclusiones}

El presente estudio muestra que árboles altamente visitados por adultos de $C$. welensii, y con elevada presencia de daños por grandes cerambícidos, mantuvieron una actividad fotosintética más elevada que los árboles no visitados por dicho insecto (y sin signos aparentes de colonización) cerca del comienzo de su actividad de vuelo diario (crepúsculo). En dicho grupo de árboles (con C. welensii) se había encontrado una presencia relevante de limoneno en la composición de la emisión foliar. Por lo tanto el comportamiento fisiológico encontrado podría haber favorecido la emisión foliar de algunos compuestos, en particular aquellos menos dependientes de factores como la radiación existente o la temperatura y menos volátiles, condiciones ambas que cumple el limoneno en contraste con los compuestos de tipo pineno. Estos resultados tienen interés para comprender las interacciones planta -insecto, especialmente las que afectan a la selección de hospedantes por el insecto xilófago $C$. welensii, que podrían tener aplicación en el manejo integrado de plagas de este cerambícido. Se requiere más investigación para analizar el modo en que cambios en la actividad fisiológica de $Q$. suber (debido a daños por $C$. welensii y/o al estrés ambiental) influyen tanto en el comportamiento emisor de los árboles como en su idoneidad para el desarrollo de progenies de C. welensii.

\section{Agradecimientos/Financiación}

Agradecemos a Sebastiana Malia, Agustín Rincón y María del Mar González por su ayuda tanto en el trabajo de campo como en el laboratorio, y al doctor Manuel Fernández por sus constructivos comentarios.

\section{Bibliografía}

Aronson, J.; Pereira, J.S.; Pausas, J.G.; (eds.), 2009. Cork oak woodlands on the Edge. Ecology, Adaptive Management, and Restoration. Society for Ecological Restoration International, Island Press, Washington.

Allison, J.D.; Borden, J.H.; Seybold, J.H.; 2004. A review of the chemical ecology of the Cerambycidae (Coleoptera). Chemoecol. 14: 123-150. https://doi.org/10.1007/s00049-0040277-1

Bates, D.; Maechler, M.; Bolker, B.; Walker, S.; 2014. Ime4: Linear mixed-effects models using Eigen and S4. R package version 1.1-7. http://CRAN.R-project.org/package=lme4>.

Benjamini, Y.; Hochberg, Y.; 1995. Controlling the false discovery rate: a practical and powerful approach to multiple testing. J. R. Stat. Soc. Ser. B57: 289-300. https://doi.org/ 10.1111/j.2517-6161.1995.tb02031.x 
Comisión Europea, 2008. El Plan de Acción de La Unión Europea en Favor de la Biodiversidad. Detener la pérdida de biodiversidad para 2010 - y más adelante. Oficina de Publicaciones Oficiales de las Comunidades Europeas, Luxemburgo. 28 pp.

Comunidad Autónoma de Andalucía, 2010. Ley 7/2010, de 14 de julio, para la Dehesa. «BOJA» núm. 144, de 23 de julio de 2010. «BOE» núm. 193, de 10 de agosto de 2010.

Consejo de las Comunidades Europeas, 1992. Directiva 92/43/EEC del Consejo, de 21 de mayo de 1992, relativa a la conservación de los hábitats naturales y de la fauna y flora silvestres.

Correia, B.; Rodriguez, J.L.; Valledor, L.; Almeida, T.; Santos, C.; Cañal, M.J.; Pinto, G.; 2014. Analysis of the expression of putative heat-stress related genes in relation to thermotolerance of cork oak. J. Plant. Physiol. 171: 399-406. https://doi.org/10.1016/j.jplph.2013.12.004

Delfine, S.; Csiky, O.; Seufert, G.; Loreto, F.; 2000. Fumigation with exogenous monoterpenes of a non-isoprenoid-emitting oak (Quercus suber): Monoterpene acquisition, translocation, and effect on the photosynthetic properties at high temperatures. New Phytol. 146: 27-36. https://doi.org/10.1046/j.1469-8137.2000.00612.x

Google, 2007. Satellite image of Dehesa San Enrique (37'15'43.73"N, 6²8'34.65"O, $80 \mathrm{~m}$ asl). Almonte, Huelva, Spain. October 2015. http://earth.google.com

Grote, R.; 2007. Sensitivity of volatile monoterpene emission to changes in canopy structure: a model-based exercise with a process-based emission model. New Phytol. 173: 550-561. https://doi.org/10.1111/j.1469-8137.2006.01946.x

Grote, R.; Monson, R.; Niinemets, Ü.; 2013. Leaf-level models of constitutive and stress-driven volatile organic compound emissions. In: Niinemets, Ü., Monson, R.K. (eds.), Biology, controls and models of tree volatile organic compound emission. Springer Netherlands, Dortrecht. pp. 315-355. https://doi.org/10.1007/978-94-007-6606-8_12

Hakola, H.; Laurila, T.; Lindfors, V.; Helleén, H.; Gaman, A.; Rinne, J.; 2001. Variation of the VOC emission rates of birch species during the growing season. Boreal. Environ. Res. 6: 237-249.

Hoeber, S.; Leuschner, C.; Köhler, L.; Arias-Aguilar, D.; Schuldt, B.; 2014. The importance of hydraulic conductivity and wood density to growth performance in eight tree species from a tropical semi-dry climate. For. Ecol. Manage. 330: 126-136. https://doi.org/10.1016/j.foreco.2014.06.039

Lavoir, A.V.; Duffet, C.; Mouillot, F.; Rambal, S.; Ratte, J.P.; Schnitzler, J.P.; Staudt, M.; 2011. Scaling-up leaf monoterpene emissions from a water limited Quercus ilex woodland. Atmos. Environ. 45: 2888-2897. https://doi.org/10.1016/j.atmosenv.2011.02.005

Lenth, R.V.; 2014. lsmeans: Least-Squares Means. R package version 2.10. http://CRAN.R-project.org/package $=1$ smeans

Llusià. J.; Peñuelas, J.; Asensio, D.; Munné-Bosch, S.; 2005. Airborne limonene confers limited thermotolerance to Quercus ilex. Physiol. Plant. 123: 40-48. https://doi.org/10.1111/j.13993054.2004.00426.x

Llusià, J.; Roahtyn, S.; Yakir, D.; Rotenberg, E.; Seco, R.; Guenther, A.; Peñuelas, J.; 2015. Photosynthesis, stomatal conductance and terpene emission response to water availability in dry and mesic Mediterranean forests. Trees 11 pp. https://doi.org/10.1007/s00468-015-1317$\mathrm{x}$

López-Pantoja, G.; Domínguez, L.; Sánchez-Osorio, I.; 2008. Mark-recapture estimates of the survival and recapture rates of Cerambyx welensii Küster (Coleoptera Cerambycidae) in a cork oak dehesa in Huelva (Spain). Cent. Eur. J. Biol. 3: 431-441. https://doi.org/ $10.2478 / \mathrm{s} 11535-008-0044-3$ 
Loreto, F.; Ciccioli, P.; Cecinato, A.; Bracaleoni, E.; Frattoni, M.; Tricoli, D.; 1996. Influence of environmental $\alpha$-pinene from factors and air composition on the emission of Quercus ilex leaves. Plant. Physiol. 110: 267-275. https://doi.org/10.1104/pp.110.1.267

Loreto, F.; Pollastri, S.; Fineschi, S.; Velikovac, V.; 2014. Volatile isoprenoids and their importance for protection against environmental constraints in the Mediterranean area. Environ. Exp. Bot. 103: 99-106. https://doi.org/10.1016/j.envexpbot.2013.09.005

Millar. J.G.; Hanks, L.M.; 2017. Chemical ecology of cerambycid beetles. In: Wang, Q., (ed.), Cerambycidae of the world: biology and management. Boca Raton: CRC Press/Taylor \& Francis.

Munné-Bosch, S.; Peñuelas, J.; Asensio, D.; Llusià, J.; 2004. Airborne ethylene may alter antioxidant protection and reduce tolerance of holm oak to heat and drought stress. Plant Physiol. https://doi.org/10.1104/pp.104.050005

Niinemets, U.; Seufert, G.; Steinbrecher, R.; Tenhunen, J.D.; 2002. A model coupling foliar monoterpene emissions to leaf photosynthetic characteristics in Mediterranean evergreen Quercus species. New Phytol. 153: 257-275. https://doi.org/10.1046/j.0028-646X.2001.00324.x

Núñez, L.; Plaza, J.; Pérez-Pastor, R.; Pujadas, M.; Gimeno, B., Bermejo, V.; García-Alonso, S.; 2002. High water vapour pressure deficit influence on Quercus ilex and Pinus pinea field monoterpene emission in the central Iberian Peninsula (Spain). Atmos. Environ. 36: 44414452. https://doi.org/10.1016/S1352-2310(02)00415-6

Oksanen, F.; Blanchet, G.; Kindt, R. et al.; 2015. vegan: Community Ecology Package. R package version 2.2-1. http://CRAN.R-project.org/package=vegan

Oliveira, G.; Werner, C.; Mertens, C.; Correia, O.; 1993. Influencia de la posición de la copa sobre la fenología y las relaciones hídricas en alcornoque (Quercus suber). Actas del I. Congreso Forestal Español 1:277-282.

Pearse, I.S.; Gee, W.S.; Beck, J.J.; 2013. Headspace Volatiles from 52 oak species advertise induction, species identity, and evolution, but not defense. J. Chem. Ecol. 39: 90-100. https://doi.org/10.1007/s10886-012-0224-5

Peñuelas, J., Llusià, J., 2002. Linking photorespiration, monoterpenes and thermotolerance in Quercus. New Phytol. 155: 227-237. https://doi.org/10.1046/j.1469-8137.2002.00457.x

Peñuelas, J.; Llusia, J.; 1999. Seasonal emission of monoterpenes by the Mediterranean tree Quercus ilex in field conditions: Relations with photosynthetic rates, temperature and volatility. Physiol. Plantarum 105: 641-647. https://doi.org/10.1034/j.1399-3054.1999.105 407.x

Peñuelas, J.; Llusià, J.; Asensio, D.; Munné-Bosch, S.; 2005. Linking isoprene with plant thermotolerance, antioxidants and monoterpene emissions. Plant. Cell. Environ. 28: 278-86. https://doi.org/10.1111/j.1365-3040.2004.01250.x

Piayda, A.; Dubbert, M.; Rebmann, C.; Kolle, O.; Costa, Silva, F.; Correia, A.; Pereira, J.S.; Werner, C.; Cuntz, M.; 2014. Drought impact on carbon and water cycling in a Mediterranean Quercus suber L. woodland during the extreme drought event in 2012. Biogeosciences 11: 7159-7178. https://doi.org/10.5194/bg-11-7159-2014

Pio, C.A.; Silva, P.A.; Cerqueira, M.A.; Nunes, T.V.; 2005. Diurnal and seasonal emissions of volatile organic compounds from cork oak (Quercus suber) trees. Atmos. Environ. 39: 1817-1827. https://doi.org/10.1016/j.atmosenv.2004.11.018

Sánchez-Osorio, I.; López-Pantoja, L.; Tapias, R.; Pareja-Sánchez, E.; Domínguez, L.; 2019. Monoterpene emission of Quercus suber L. highly infested by Cerambyx welensii Küster Ann. For. Sci. 89. https://doi.org/10.1007/s13595-019-0879-y 
Tenhunen, J.D.; Reynolds, J.F.; Lange, O.L.; Dougherty, R.L.; Harley, P.C.; Kummerow, J.; Rambal, S.; 1989. QUINTA: a physiologically based growth simulator for drought adapted woody by mediterranean sclerophyll shrubs during summer drought. In: Pereira, J.S.; Landsberg, J.J.; (eds.), Biomass. NATO ASI series, Applied Science, Vol. 166. Dordrecht, The Netherlands: Kluwer, 135-68. https://doi.org/10.1007/978-94-009-2348-5_9

Torres-Vila, L.M.; Mendiola-Díaz, F.J.; Sánchez-González, Á.; 2017. Dispersal differences of a pest and a protected Cerambyx species (Coleoptera: Cerambycidae) in oak open woodlands: a mark-recapture comparative study. Ecol. Entomol. 42: 18-32. https://doi.org/10.11 11/een.12355

Vaz, M.; Pereira, J.S.; Gazarini, L.C.; David, T.S.; David, J.S.; Rodrigues, A.; Maroco, J.; Chaves, M.M.; 2010. Drought-induced photosynthetic inhibition and autumn recovery in two Mediterranean oak species (Quercus ilex and Quercus suber). Tree Physiol. 30: 946-956. https://doi.org/10.1093/treephys/tpq044

Vaz, M.; Maroco, J.; Ribeiro, N.; Gazarini, L.C.; Pereira, J.S.; Chaves, M.M.; 2011. Leaf-level responses to light in two co-occurring Quercus (Quercus ilex and Quercus suber): leaf structure, chemical composition and photosynthesis. Agroforest. Syst. 82:173-181. https://doi.org/10.1007/s10457-010-9343-6

Vicente, C.; 2013. Evaluación del grado de afectación por larvas de Cerambyx welensii Küster 1846 (Col., Cerambycide) del arbolado de las dehesas (Quercus suber, L y Q. rotundifolia, Lam.) en Extremadura y su relación con algunas variables importantes del ecosistema. Doctoral Thesis, University of Extremadura, Badajoz, Spain .

WWF/Adena, 2006. La dehesa en los Programas de desarrollo rural 2007-13. Propuesta. 34 pp. 